EchoGéo

ECHOGEO-Sur le Vif | 2013

\title{
Les ressorts de l'intervention militaire tchadienne au Mali (2013)
}

\section{Géraud Magrin}

\section{OpenEdition}

Journals

Édition électronique

URL : http://journals.openedition.org/echogeo/13444

DOI : $10.4000 /$ echogeo.13444

ISSN : 1963-1197

Éditeur

Pôle de recherche pour l'organisation et la diffusion de l'information géographique (CNRS UMR 8586)

\section{Référence électronique}

Géraud Magrin, «Les ressorts de l'intervention militaire tchadienne au Mali (2013)», EchoGéo [En ligne], Sur le Vif, mis en ligne le 28 juin 2013, consulté le 30 avril 2019. URL : http:// journals.openedition.org/echogeo/13444; DOI : 10.4000/echogeo.13444

Ce document a été généré automatiquement le 30 avril 2019

\section{(c) (i) (9)}

EchoGéo est mis à disposition selon les termes de la licence Creative Commons Attribution - Pas d'Utilisation Commerciale - Pas de Modification 4.0 International 


\title{
Les ressorts de l'intervention militaire tchadienne au Mali (2013)
}

\author{
Géraud Magrin
}

Le 11 janvier 2013, l'armée française intervenait au Mali pour stopper une offensive de forces hétéroclites abritées derrière l'étendard islamiste (Aqmi, Mujao, Ansar Eddine ${ }^{1}$ ) qui avaient pris le contrôle du Nord en mars $2012^{2}$ et menaçaient le fragile équilibre politique installé depuis à Bamako. Une semaine plus tard seulement, avant même que les forces africaines sous-régionales organisées dans le cadre de la Misma (Mission des Nations unies au Mali) commencent un déploiement timide dans les grandes villes du sud du Mali, 1400 soldats tchadiens entraient au Mali par l'Est, après avoir traversé le Niger au nord du lac Tchad - avant garde d'un contingent promis de 2000 hommes. Ils rejoignaient aussitôt l'armée française qui, après la prise rapide des villes méridionales du Septentrion malien (Tombouctou, Gao, Kidal), se dirigeait vers l'Adrar des Ifoghas, où subsistaient les principales bases et concentrations des forces ennemies. Là, les troupes françaises et tchadiennes menaient ensemble de durs combats. Le 2 et 3 mars, à l'annonce de lourdes pertes dans les rangs tchadiens (une vingtaine de morts et autant de blessés), répondait la revendication par l'armée tchadienne de la mort de deux des principaux leaders islamistes (Abou Zeid (Aqmi) et Mokhtar Belmokhtar (proche du Mujao)). Au moment où nous écrivons ces lignes (juin 2013), cette guerre est entrée dans une phase de conflit asymétrique: l'armée malienne et ses alliés (français et africains) sont soumis à des attaques sporadiques et à des attentats, au Mali et dans les pays voisins ${ }^{3}$; la France et le Tchad ont entamé le retrait de leurs troupes en avril 2013. Celles-ci doivent être remplacées par une force onusienne appelée à montée en puissance ; la mort de Mokhtar Belmokhtar a été démentie.

2 Ce que nous voulons ici comprendre, ce sont les ressorts de l'intervention tchadienne au Mali aux côtés de l'armée française, dans une configuration apparemment inédite. Ce pays non membre de la Cedeao (Communauté économique des États de l'Afrique de l'Ouest ${ }^{4}$ ) intervient seul aux côtés de la France, massivement et en première ligne, dans un pays qui ne lui est pas riverain et est situé à $2000 \mathrm{~km}$ de ses frontières. Si l'ampleur de l'engagement de la France (déploiement de plus de 3500 militaires au sol et mobilisation 
d'importants moyens aériens) est nouvelle dans ce pays, qui n'avait pas connu semblable déstabilisation entre l'indépendance et mars $2012^{5}$, elle se comprend assez bien: les progrès des différents groupes islamistes radicaux s'en prenant aux intérêts français dans le Sahara (enlèvement et assassinats de Français en Mauritanie, au Niger, au Mali depuis le début de la décennie 2000 (voir Grégoire, Bourgeot, 2011 et Bourgeot, 2011)) menaçaient à présent l'existence même de cet État, dont la fragile vitrine démocratique avait été pulvérisée en mars 2012 (Bayart, 2013a). Au-delà, ils mettaient en péril les équilibres politiques de pays sahéliens avec lesquels la France partage une communauté d'intérêts (culturels, migratoires) qui dépassent des enjeux économiques et stratégiques limités pour l'essentiel au contrôle de l'uranium du Niger par Areva. En outre, le cadre diplomatique mis en place au cours des mois précédents au service d'un projet légèrement différent (Bayart, 2013b) a coupé court aux débats sur la légitimité internationale d'une telle action.

Mais que viennent faire les soldats tchadiens au Mali? Le principal argument avancé par le pouvoir tchadien est la lutte contre le terrorisme islamiste, qui constituerait une menace pour toute cette partie de l'Afrique ${ }^{6}$. Cette raison est au mieux secondaire selon nous. Malgré la proximité géographique de la crise liée au mouvement Boko Haram au nord-est du Nigeria et l'instabilité croissante de la Libye post-Kadhafi, le Tchad semble pour le moment à l'abri d'une déstabilisation de ce type. Nous voudrions montrer ici que l'intervention militaire franco-tchadienne au Mali révèle avant tout un moment de l'État tchadien caractérisé par l'hybridation d'un ethos guerrier construit dans l'histoire (précoloniale, coloniale et contemporaine) (Reyna, 1990; Bégin-Favre, 2008) - ou la trajectoire historique d'un État gouverné par la violence (Debos, 2009; 2013a) - et de l'influence de la situation pétrolière qui prévaut depuis 2003 (Soares de Oliveira, 2007; Magrin, 2013), qui permet l'affirmation d'un leadership régional et dessine une figure originale d'insertion dans la mondialisation. Alors que l'appui de la France a, en 2006 et 2008, sauvé in extremis le pouvoir tchadien menacé de rébellions (Magrin, 2008), notre hypothèse est que l'intervention tchadienne au Mali vise à la fois l'actualisation d'une rente géopolitique externe (une nouvelle «dette du sang » vis-à-vis de la France) et la gestion d'équilibres qui relèvent de la scène politique intérieure. Le risque pour le Tchad, au-delà du coût financier et humain de l'opération, étant que cette dynamique sape les fragiles efforts de construction d'un État développementaliste auxquels on avait assisté depuis le retour de la paix dans le pays, en 2009.

\section{Le « métier des armes » au Tchad : une longue histoire}

4 L'intervention militaire tchadienne au Mali s'inscrit dans une histoire longue où le «métier des armes» (Debos, 2009; 2013a) occupe une place centrale, notamment dans certains groupes de la population qui constituent la base du pouvoir actuel. Dans cette partie enclavée de l'Afrique qu'est le cœur du bassin du lac Tchad, des royaumes précoloniaux à aujourd'hui, de manière continue malgré des formes différentes selon les régions et les moments, le métier des armes a constitué un statut social et un moyen central d'accéder au pouvoir et à la richesse (Debos, 2009).

5 L'image de l'intervention tchadienne rendue aujourd'hui par les médias qui couvrent la guerre du Mali s'inscrit dans un type bien identifié depuis des décennies en France, où il exerce notamment une fascination dans l'armée, chez certains journalistes, voire au sein du grand public. Ce type rend compte à la fois d'une forme originale de pratique militaire 
et d'une esthétique qui y est associée : les "guerriers du désert » intrépides, efficaces et esthétiques (treillis turban lunettes noires), spécialistes du "rezzou TGV », qui désigne une attaque menée par une colonne de toyotas équipées de mitrailleuses lancées à pleine vitesse. Cette technique a été popularisée au moment des guerres tchado-libyennes des années 1980 menées pour l'essentiel dans le Sahara, où des groupes de combattants principalement originaires de ces régions sont chargés sur le plateau arrière de pick up toyota équipés de mitrailleuses, aujourd'hui souvent garnis de chapelets de roquettes, en plus des fûts d'eau et de carburant, et lancés à pleine vitesse à travers dunes et rochers (voir par exemple Darcourt, 1999). En permettant de défaire l'armée libyenne ${ }^{7}$, pourtant très supérieure au plan de l'armement conventionnel et des effectifs engagés, cette forme de combats a suscité étonnement et admiration en occident, notamment parmi les militaires. Elle a été ensuite la forme dominante des nombreux conflits internes qui ont émaillé la décennie 2000 (Magrin, 2008), même si les avions, hélicoptères et chars dont la rente pétrolière a permis l'achat au gouvernement ont quelque peu changé la donne à partir de 2008. Ces conflits, en opposant troupes gouvernementales et rebelles (souvent originaires des mêmes ethnies ou de groupes proches) cachées dans les montagnes du Tibesti ou de l'Ennedi, à la frontière du Darfour, présentent des théâtres fort analogues à ceux de l'Adrar des Ifoghas des combats de 2013. Si les soldats tchadiens sont au Mali, c'est bien en partie parce qu'ils ont l'expérience du combat dans le désert. Mais cela n'est pas une explication suffisante. L'armée tchadienne présente au Mali et les représentations qui en sont données sont le produit d'une histoire longue.

6 L'histoire médiévale et moderne du Tchad peut être décrite en grande partie à travers celle de royaumes musulmans centralisés tirant leur pouvoir du contrôle du commerce à longue distance entre le monde méditerranéen et le sahel. Parmi les principaux «produits » échangés figuraient les esclaves, prélevés sur les marges méridionales non islamisés par des razzias incessantes et les armes importées. Il s'ensuivait des « guerres sans fin » (Reyna, 1990) entre royaumes rivaux, ceux-ci étant des structures militarisées et violentes (Reyna, 1990; Bégin-Favre, 2008). La colonisation brisa ces structures politiques, tout en réorientant l'économie et les réseaux d'échanges vers le sud. La fonction militaire garda cependant une place centrale au Tchad : au nord, le recrutement des auxiliaires et autres méharistes perpétua un horizon militaire dans le cadre du maintien de l'ordre au sein du territoire colonial (Debos, 2009). Mais c'est surtout au sud (et au Guéra), régions plus densément peuplées et aux populations perçues comme plus disciplinées, que seront recrutés un grand nombre de «tirailleurs sénégalais » lors des deux guerres mondiales.

7 Peu après l'indépendance, où des originaires du Sud Sara, plus éduqués, prennent le pouvoir, une rébellion éclate et s'étend rapidement aux régions musulmanes du pays (Sahel et Sahara). Ce moment ouvre une période pratiquement ininterrompue, de 1966 à 2009, où des rébellions, installées sur les différentes périphéries du territoire, mobilisées sur une base ethno-régionale autour de leaders qui s'imposent avant tout par leurs talents guerriers - les politistes parlent de factionnalisme (Lemarchand, 1986; Buijtenhuis, 1987) -, se battent contre le pouvoir en place. En 1979, le pouvoir échappe aux originaires du Sud. Ceux-ci alimenteront quelques rébellions dans les années 1980 et 1990. Hissein Habré, un Toubou de Faya ${ }^{8}$, prend le pouvoir en 1982. Des luttes entre factions et contre la Libye émailleront sa dictature. En 1990, il est chassé par un de ses principaux lieutenants, Idriss Déby, commandant de l'armée tchadienne lors des guerres contre la Libye, qui prend le pouvoir à l'issue d'un raid de $900 \mathrm{~km}$ à partir de la frontière 
soudanaise en 1990. Au cours des années 1990 et 2000, le pouvoir d'Idriss Déby est régulièrement contesté par les armes. La vie politique du pays s'organise au gré de cycles de rébellion, répression, réconciliation (Magrin, 2008).

8 Au gré de trajectoires fluides (Debos, 2008), les trajectoires des chefs et des simples combattants ne sont pas les mêmes: les premiers passent du statut de chefs rebelles à celui de ministres, ou sont intégrés dans l'armée à des grades d'officiers supérieurs, et inversement; les seconds peuvent aussi changer de statut - d'une rébellion à l'armée régulière ou à la douane, ou à la fonction de coupeur de route quand l'absence de leader ou les rencontres de circonstance le permettent (Saibou, 2010), mais ils connaissent des possibilités d'ascension sociale plus aléatoires (Debos, 2008). Pour tous, la mort violente est un horizon toujours proche, un risque assumé. Le recrutement privilégié, quoique non exclusif, de ces combattants au sein des régions du nord et du nord-est du pays, s'explique aussi par la marginalisation de ces espaces durant les deux premiers tiers du $\mathrm{XX}^{\mathrm{e}}$ siècle (Bégin-Favre, 2008) : les taux de scolarisation y ont été historiquement faibles - le refus de l'école (Khayar, 1976) ayant constitué une forme de repli face à l'acculturation liée au modèle colonial -, les investissements de l'État, colonial puis indépendant, se sont concentrés dans le sud (Magrin, 2001), les sécheresses des années 1970-1980, les conflits ultérieurs et l'instrumentalisation des lignes de clivage entre groupes par l'État au cours des conflits des années 2000 n'ont rien arrangé (Bégin-Favre, 2008).

9 La question de la place de la jeunesse dans une Afrique en crise politico-économique dans un contexte de croissance démographique sans précédent, qui a servi de toile de fond à bien des conflits des années 1990-2000 (rôle des enfants soldats en Sierra Leone et au Liberia (Richards, 1996), au Congo (Bazenguissa-Ganga, 1996), en RDC (Vlassenroot, 2006), en Côte d'Ivoire (Chauveau, 2005), etc.), se pose au Tchad comme ailleurs. Dans ce pays, 300000 jeunes arrivent chaque année sur un marché du travail (Guengant, 2012) où l'agriculture et l'élevage, qui fournissent encore $80 \%$ de la population active, ne fournissent pas des horizons très accueillants. Cette question de l'intégration sociale des jeunes s'intègre ici moins dans le cadre de conflits entre aînés et cadets, comme en Afrique de l'Ouest, que dans la perspective des conséquences de la marginalisation historique de ces régions sahélo-sahariennes du Tchad, peu favorable à la modification de traits culturels hérités : pour les populations peu ou non scolarisées d'origine saharienne (Toubou, Zaghawa, parfois Arabes), le courage est une vertu supérieure, la mort un risque assumé, la violence contre les ennemis du clan licite, tout comme la razzia, qui montrent l'habileté à survivre dans un environnement hostile. L'incapacité de l'État colonial puis indépendant à dessiner des perspectives de développement dans ces régions du Nord et de l'Est du Tchad, que les conflits successifs ne suffisent pas à expliquer, contribue à la permanence de cet ethos guerrier au sein de ces sociétés. Pour de nombreux jeunes de l'Est ou du Sahara tchadien sans qualification, le métier des armes apparait finalement comme une option aussi raisonnable que d'autres dans des contextes incertains - la voie idéale conduisant de la rébellion à une place de soldat dans la capitale. Ce statut est relativement envié et socialement valorisé comme figure du pouvoir, car il entre en résonance avec les codes sociaux. Il donne aussi accès à des espoirs d'ascension sociale, même si les opportunités d'accumulation réelles demeurent rares et sélectives. Entendons-nous bien: nous ne postulons pas l'existence d'un lien de causalité entre un ethos guerrier intemporel, a-historique, et le rapport contemporain de ces sociétés à la violence et aux armes. Ces aspects culturels issus de l'histoire sont un registre dans lequel les acteurs contemporains sont libres de puiser, ou non, selon les circonstances. Or, le 
fonctionnement de l'État et du politique au Tchad au cours des dernières décennies ont été favorables à leur mobilisation.

En effet, depuis les années 1980, le basculement du pouvoir au Nord s'est manifesté par le prisme de cette situation politique où la violence permet l'accumulation et légitime le pouvoir, jusqu'à son sommet. La démocratisation formelle, consentie sous pression occidentale dans le contexte des années 1990, n'a paru pouvoir menacer le régime qu'au moment de la Conférence nationale souveraine de 1993-96. Ensuite, la légitimation par les armes a pris le pas sur les urnes - l'organisation d'élections présidentielles largement contrôlées par le pouvoir (en 1996, 2006, 2011) ayant comme double sens de satisfaire les partenaires occidentaux et de constituer des moments de mobilisation clientéliste et de redistribution. La maîtrise du pouvoir réside ainsi dans la capacité à organiser à son profit cette scène politico-militaire instable, que les soutiens étrangers successifs (venant de Libye ou du Soudan) rendent particulièrement dangereux.

11 La participation de Tchadiens aux conflits de la sous-région participe de cette logique. Elle s'inscrit tantôt dans les stratégies de l'État, tantôt dans celles d'individus, marquant le débordement $\mathrm{du}$ factionnalisme tchadien sur une scène régionale élargie. Ainsi, de nombreux Toubou tchadiens ont, après la défaite de Goukouni Oueddeï en 1982 face à Hissein Habré, rejoint l'armée libyenne, où certains sont devenus officiers supérieurs. À la fin des années 1990, un contingent de l'armée tchadienne est intervenu en RDC, à la demande libyenne, aux côtés du président Kabila contre les rébellions de Jean-Pierre Bemba, soutenues par l'Ouganda - connaissant un grave revers9 attribué par les médias aux conditions environnementales très éloignées de celles du sahel tchadien, ce déterminisme climatique devant sans doute être tempéré par la référence à l'étirement des lignes de communication et à la difficulté d'assurer la logistique sur des milliers de kilomètres sans infrastructures. Au cours des années 2000, le conflit du Darfour soudanais est entré en résonnance avec les crises politiques nationales, un système d'alliances croisées favorisant l'enrôlement d'un nombre conséquent de Zaghawas tchadiens aux côtés des rebelles darfouri (certains restant fidèles au président Déby) (Marchal, 2006; Tubiana, 2011). Des combattants tchadiens sont surtout intervenus à plusieurs reprises en RCA, participant à la $\mathrm{MISAB}^{10}$ à la fin des années 1990, puis surtout appuyant les « libérateurs » de François Bozizé en 2003. Alors que des combattants tchadiens fidèles à des seigneurs de guerre comme Abdoulaye Miskine entretenaient l'insécurité dans le Nord de la RCA, l'armée tchadienne apparait, jusqu'aux récents combats de décembre 2012, comme le principal rempart du régime de François Bozizé contre les rébellions successives qui le contestent (voir ICG, 2007). Le Tchad, sur un mode comparable à celui de la Libye du colonel Kadhafi, mais mineur, contribue à nourrir des conflits régionaux tout en faisant figure de pôle stabilisateur (Marchal, 2013). Ainsi, comme d'autres régions pauvres dans l'histoire, l'exportation de combattants, mobilisés selon des registres variés (via l'armée régulière, derrière un seigneur de guerre, ou à travers des stratégies plus individuelles), constitue une des formes d'insertion du Tchad au sein d'un des carrefours africains les plus troublés au cours du demi siècle écoulé.

\section{Le Tchad et la France : le retour de flamme d'une longue fraternité d'armes}

L'intervention tchadienne au Mali est aussi le produit d'une relation franco-tchadienne au long cours, dont la dimension militaire est plus importante qu'ailleurs. Le Tchad 
résonne ainsi d'une manière particulière dans l'imaginaire militaire français (Tulipe, 2008).

L'attrait exercé par le Sahara sur les militaires français a sa part dans cette relation particulière avec le Tchad. Ils y trouvèrent un espace d'aventure et d'autonomie relative au moment de la conquête, puis un milieu dont la configuration géographique favorisait leur pouvoir par rapport à celui de l'administration civile : primat du contrôle stratégique sur le développement économique dans l'immensité du désert.... Le Tchad resta territoire militaire jusqu'en 1920 (alors que le Sénégal était une colonie depuis 1889); et l'armée française continua d'administrer les préfectures sahariennes du Borkou Ennedi Tibesti cinq ans après l'indépendance, en 1965 (Caron, 2009). Les régiments de méharistes ont joué un rôle particulier dans cette histoire, popularisée à travers la littérature, comme les romans de Joseph Peyré11 ${ }^{11}$ ou à travers les récits d'anciens méharistes (voir par exemple Garbit, 1996; Besnier, 2011). Ceux-ci ne se déroulent pas uniquement au Tchad, mais ce pays, confronté à la menace de la confrérie senussiyya (très influente au Fezzan) jusque durant la Première Guerre mondiale, puis placé sur le front de la Seconde Guerre mondiale, y eut une place éminente. Il fut aussi la base de la France libre et de l'organisation de la colonne Leclerc, et à ce titre support de mémoire (Caron, 2005).

La France fut ensuite impliquée, à son corps plus ou moins défendant, dans la plupart des conflits post-indépendance du Tchad. La Légion étrangère fut déployée de la fin de 1969 à 1972 aux côtés de l'armée tchadienne pour contrer les progrès du Frolinat - obtenant des succès militaires sans lendemain, non sans infliger de lourds préjudices aux populations civiles (Buijtenhuis, 1978) -, avant que les opérations Manta (1983) et Épervier (1986) ne soient dirigées contre l'influence libyenne. La victoire contre la Libye de 1987 est celle de l'alliance entre la couverture aérienne et le soutien logistique français et l'efficacité des colonnes de toyotas des Tchadiens - qui ne sont pas sans évoquer l'actuelle guerre au Mali, les différences principales résidant dans le terrain (non tchadien), l'adversaire (des forces non conventionnelles aux modes d'organisation plus proches de ceux de groupes rebelles tchadiens que des régiments mécanisés de l'armée libyenne soutenue par l'URSS des années 1980), et les innovations technologiques (renseignements satellites, drones, etc.).

Après les conflits tchado-libyens, la France conserva une base permanente à N'Djaména avec environ 1000 militaires et des moyens aériens conséquents (au minimum 2 Mirages 2000, 2 transall, des hélicoptères). Quand une remise à plat de la présence militaire française en Afrique était à l'étude pour des raisons budgétaires, le contexte permettait opportunément de justifier l'utilité de cette base. Ce fut le cas d'abord face aux troubles de l'Afrique centrale (guerre et génocide au Rwanda en 1994, troubles en RCA en 1996-97) puis lors du conflit du Darfour et de ses débordements au Ouaddaï, cause d'un vaste déploiement humanitaire puis militaire international, au sein duquel la France joua un rôle important (2004-2009) ${ }^{12}$. En avril 2006 et février 2008, l'armée française joua un rôle décisif d'appui à Idriss Déby (au minimum sur le plan du renseignement et de la logistique), lorsque des colonnes de rebelles venus de la frontière soudanaise portèrent les combats jusque dans N'Djaména (Iyebi-Mandjek, 2008). Plus récemment, au moment de la guerre en Libye (2011), puis au Mali, le dispositif Épervier se révéla à nouveau fort utile. S'il est fréquemment au cœur de négociations franco-tchadiennes - concernant notamment les conditions financières de son maintien, dont les Tchadiens essaient de tirer le meilleur parti -, aucune des deux parties ne semble décidée à s'en passer. Idriss Déby, en insistant à plusieurs reprises au printemps 2013 sur le danger représenté par le 
Sud libyen, qui accueillerait à présent l'essentiel des groupes terroristes chassés de Libye, conforte ainsi la valeur stratégique du dispositif tout en présentant le Tchad comme un rempart à conforter.

16 Au-delà des raisons stratégiques et des souvenirs, d'autres considérations entrent en ligne de compte pour expliquer l'attachement militaire français au Tchad (Tulipe, 2008) : la possibilité de disposer d'un terrain d'entraînement en milieu désertique grand comme la France, le fait qu'Épervier émarge parmi les Opex (opérations extérieures), donc hors du budget de la Défense, mais aussi, sans doute, la perspective d'offrir un horizon de dépaysement, d'aventure et de solde élevée à ses militaires en contexte de professionnalisation des armées.

17 Si le Tchad résonne dans l'imaginaire militaire français, l'inverse est également vrai. La culture et l'imaginaire militaire au Tchad doivent encore aux relations avec la France, même si les anciens combattants, de moins en moins nombreux et influents, appartiennent à l'histoire : aux formations classiques accordées aux cadres de l'indépendance ont succédé des formes de coopération militaire qui essayèrent de s'adapter à l'originalité de l'armée tchadienne. Des formations à l'École de Guerre accompagnèrent des chefs rebelles devenus officiers supérieurs. Idriss Déby est diplômé de l'Institut aéronautique Armaury-La-Grange (Hazebrouck). La résilience du président tchadien réside sans doute dans sa capacité à articuler logiques militaires occidentales et registre local. Quoi qu'il en soit, le fait qu'il ait, en 2009, rebaptisé «Place de la Grande armée » l'ancienne place de l'Étoile, cœur du quartier colonial de l'ancienne Fort-Lamy, exprime non sans une certaine ironie ${ }^{13}$ les formes d'influence martiales que l'histoire de l'hexagone a exercées sur lui.

18 L'intervention franco-tchadienne au Mali s'inscrit, outre ce vieux compagnonnage d'armes, dans la volonté occidentale et africaine de mettre en place une répartition des rôles dans la gestion des crises adaptées au contexte post-guerre froide. Cette ambition remonte à la fin des années 1990, quand, face à la multiplication des conflits en Afrique et au génocide rwandais - qui traduit notamment l'articulation d'un contexte de crise économique, de démocratisation et de sevrage des rentes géopolitiques de la guerre froide -, les États-Unis, le Royaume-Uni et la France s'entendent sur le besoin de coordonner leurs appuis en matière de renforcement des capacités africaines de maintien de la paix. Le programme Recamp ${ }^{14}$ élaboré par la France en 1998, placé sous l'égide des Nations Unies et de l'Union africaine, a pour principe une contribution financière française (et, au-delà, des membres volontaires de la « communauté internationale ») à la formation et à l'équipement des armées africaines, afin que celles-ci soient en mesure d'intervenir en première ligne dans les opérations de maintien de la paix à mener en Afrique, quitte à ce que les pays occidentaux apportent un soutien logistique, en matière de renseignement, voire de couverture aérienne, selon les nécessités propres à chaque théâtre. On se situe à l'amont de la tentation contemporaine des guerres à zéro mort (occidentaux), dont l'utilisation croissante des drones par l'Amérique d'Obama est le symptôme le plus visible. Cette idée est en outre convergente avec le souhait de l'Union africaine d'accroître le rôle des institutions africaines dans la résolution des problèmes du continent.

19 Dans la crise malienne, cependant, la répartition des rôles n'a pas été exactement celle-là. Au cours de l'année 2012, le Tchad manifestait son ouverture de principe à participer à une force africaine régionale, mais il n'entendait pas combattre seul en première ligne, voire s'il devait se trouver au milieu d'une coalition hétérogène de pays de la Cedeao aux 
capacités militaires sahariennes hypothétiques (Baruch, 2013). Si nous ne connaissons pas la nature des discussions entre autorités politiques et militaires françaises et tchadiennes qui ont entraîné l'engagement tchadien, il est manifeste que l'engagement de troupes françaises au sol a servi de déclencheur, plus près de l'ancienne fraternité d'armes que de l'esprit de Recamp... Mais au moins, l'armée de l'ancienne puissance coloniale n'est pas seule. L'esprit de la participation africaine est préservé. Au sein de l'armée française, ce conflit représenterait aussi une revanche de l'armée de terre, sur un terrain et avec des alliés bien connus, sur un conflit afghan où la dépendance envers l'armée américaine avait été une source de frustration et d'échec (Guibert, 2013). Le bilan humain après près de 8 semaines de combat- 3 tués côté français, 26 morts et une cinquantaine de blessés côté tchadien (Huffingtonpost, 2013) - suggère cependant aussi bien l'asymétrie de cette relation que des manières différentes de faire la guerre, qui traduisent le rapport différent à la mort dans les deux pays.

\section{La place des armes dans le Tchad pétrolier contemporain}

20 L'ère pétrolière inaugurée par la mise en exploitation des gisements de Doba en 2003 s'est insérée dans cette histoire fortement influencée par les fonctions militaires, mais elle en a aussi esquissé des possibilités de changement.

21 L'arrivée de la rente pétrolière fin 2003 a précédé de peu une recrudescence des conflits politico-militaires tchadiens, sur un mode connu par le passé mais dramatisé par le contexte : des proches du régime mécontents de la décision d'I. Déby de modifier la constitution pour se représenter aux élections de 2004, alors que l'arrivée de la rente aiguisait tous les appétits, sont entrés en rébellion, avec le soutien du Soudan. Les deux pays ont disposé de ressources pétrolières importantes, dans un contexte de haut niveau des cours, pour financer à la fois des groupes rebelles dans l'autre pays et acheter de l'armement. Après les durs combats de 2006 et $2008^{15}$ jusque dans les quartiers de N'Djaména, le défilé militaire d'août 2008 correspond à une démonstration de force d'un président tchadien conforté par la victoire, auquel les cours record précédant la crise financière de juillet 2008 fournissent des possibilités financières inédites. Le Tchad utilise cette année-là $50 \%$ de sa rente pétrolière en achat d'armes (Maoundonodji, 2009) et confirme les pronostics des tenants de la «malédiction pétrolière » : la rente semble bien favoriser les «États faillis durables» (Soares de Oliveira, 2007), qui rassemblent tous les travers des États post-coloniaux et pétroliers, mais disposent grâce aux hydrocarbures de moyens de coercition illimités et d'un motif permanent de légitimation externe ${ }^{16}$. La communication présidentielle de cette période proclame sans retenue le triomphe du président et la nature de son pouvoir, qui repose sur ses vertus guerrières. En un réflexe obsidional rétrospectif, N'Djaména est entourée d'un système défensif composé d'un large fossé (anti-toyota) et d'un talus, censés empêcher l'entrée de colonnes de pick-up rebelles.

Après 2008, la situation change sensiblement. Débarrassé de menace militaire directe ${ }^{17}$, le pouvoir d'Idriss Déby semble tenté d'emprunter des formes développementalistes nouvelles (Magrin, 2013). Après des décennies de conflit et d'ajustement structurel, où la sphère du développement avait été de fait monopolisée par les grands bailleurs internationaux (Banque mondiale et Union européenne en tête) ou de grandes ONG, on assiste à un retour de politiques publiques (infrastructures socio-économiques et 
sanitaires, transports, aménagement urbain et du territoire, agriculture, environnement, etc.). Certes, celles-ci rencontrent de nombreuses difficultés, tant les capacités de conception et de coordination de l'État tchadien sont exsangues, et parce que les choix réalisés reflètent aussi des préoccupations politiciennes et un mode de fonctionnement clientéliste que l'on observe au même moment dans un certain nombre de pays africains, comme au Sénégal (de Jong et Foucher, 2010). Certes, une certaine brutalité n'en est pas absente, comme lors des "déguerpissements" urbains nécessaires aux projets présidentiels à N'Djaména, ou à l'occasion de mesures destinées à protéger l'environnement, qui favorisent le renforcement des contrôles sur les ruraux (Magrin, 2013). Mais le treillis cède la place au costume trois pièces à la coupe moderne. Le discours se modernise, faisant par exemple une large place aux enjeux du développement durable. Le Tchad se positionne dans le projet panafricain de la Grande muraille verte dont le Secrétariat panafricain est installé à N'Djaména - et porte la mobilisation régionale et internationale pour le sauvetage du lac Tchad (voir Passages, 2011).

Pourquoi le Tchad s'est-il alors engagé au Mali? Le risque islamiste au Tchad semble plutôt moins fort que dans d'autres pays sahélo-sahariens comme la Mauritanie ou même le Niger. Des ONG islamistes ont bien essaimé dans le pays à la faveur de la libéralisation politique des années 1990 (Mahamat Adoum Doutoum, 1994), mais la nature autoritaire du régime les a jusqu'à présent contenues. Des conflits intercommunautaires ont eu lieu ici et là, parfois amplifiés par la radicalisation religieuse; ils ont pris de l'ampleur en particulier dans le Sud pétrolier (voir Magrin, 2001) et au Ouaddaï au milieu des années 2000 (Bégin-Favre, 2008), mais ils ont été contenus et ont perdu ensuite de leur intensité. En un mot, l'islamisme n'a guère pris de forme violente et politique. À $300 \mathrm{~km}$ de N’Djaména, la capitale de l'État du Bornou, Maïduguri, est déstabilisée depuis 2009 par un conflit sanglant opposant la secte Boko Haram à l'État nigérian (Hervieu, 2013). Dans ce bassin du lac Tchad où les circulations sont nombreuses entre populations proches, le risque d'un déplacement de l'insécurité sur le territoire tchadien n'est pas nul (Musila, 2012) - et les autorités tchadiennes ont pris des mesures préventives importantes après leur engagement militaire au Mali - il s'agit d'empêcher d'éventuelles représailles. De fait, le phénomène de Boko Haram, complexe, relève de l'imbrication de jeux de pouvoirs régionaux et nationaux propres à la scène nigériane : un débordement au Nord Cameroun ou au Tchad est hautement improbable (Saibou, 2013); il ne saurait donc justifier l'engagement militaire tchadien au Mali. De plus, la position du Tchad au centre Est de l'espace saharien l'a tenu jusqu'ici en marge des principaux flux de drogue et d'armes qui reliaient le golfe de Guinée à la méditerranée via la Mauritanie, le Mali, le Niger (Scheele, 2011), si bien qu'il a longtemps semblé le moins exposé de cet ensemble au risque terroriste. Cependant, l'instabilité persistante de la Libye post Kadhafi, le déplacement des groupes islamistes du Mali vers le Sud libyen et la fragilité structurelle du Sahara tchadien (voir ICG, 2011a et 2011b) sont porteurs de risques non négligeables pour l'avenir.

24 La crise malienne offre en fait au pouvoir tchadien une occasion inespérée, de nature très contextuelle. Au plan interne, la rapidité et les succès de l'intervention ont été d'abord plutôt bien perçus, comme l'a montré l'unanimité du soutien de l'Assemblée nationale (Hicks, 2013). Puis les pertes enregistrées, le changement de nature du conflit - d'une offensive à une lutte antiguérilla - de même que ses coûts financiers ont soulevé des interrogations dans la presse nationale, coïncidant peu ou prou avec l'annonce du retrait de l'essentiel des forces tchadiennes, à la mi-avril 2013. Les grands panneaux accueillant 
le visiteur à l'aéroport de N'Djaména, montrant Idriss Déby triomphant au milieu de ses soldats, treillis et symbole de commandement à la main (fouet en cuir d'hippopotame ?), sous un texte remerciant les Fatim (Forces armées tchadiennes d'intervention au Mali), dignes fils de l'Afrique et du Tchad, pour leurs sacrifices, n'est pas sans évoquer le registre martial de la communication déployée après les événements de février 2008, sous la tonalité plus joyeuse d'une guerre victorieuse à moindre frayeur et coût humain. La localisation de ces panneaux à l'aéroport interroge néanmoins: s'ils magnifient le triomphe du prince auprès de l'opinion nationale, nul doute qu'ils ne s'adressent aussi aux étrangers de passage.

En effet, à travers l'engagement tchadien au Mali, Idriss Déby donne l'impression de payer une dette pour le soutien français de 2008 tout en s'offrant aussi une petite revanche sur le nouveau locataire de l'Élysée. Parmi les quelques signes de "rupture " avec la présidence précédente, François Hollande avait souhaité, après son élection de mai 2012, introduire davantage d'éthique dans ses relations africaines: encourager les présidents élus démocratiquement, quel que soit leur bord politique affiché, socialistes (Alpha Condé en Guinée, Mahamadou Issoufou au Niger ${ }^{18}$ ) comme libéraux (tels Macky Sall du Sénégal, Alassane Dramane Ouattara en Côte d'Ivoire), si tant est que ces qualificatifs aient du sens sur la scène politique africaine. Inversement, F. Hollande avait publiquement battu froid plusieurs dirigeants au style plus autoritaire, réélus à maintes reprises, présidents de pays d'Afrique centrale vivant d'économies extractives. Il avait snobé Joseph Kabila au sommet de la francophonie d'octobre 2012 à Kinshasa, où il ne s'était rendu qu'in extremis, s'était gardé d'interférer avec la justice dans l'affaire des biens mal acquis impliquant les familles des présidents équato-guinéen, gabonais, congolais et tchadiens, et avait fort peu diplomatiquement tardé à rencontrer I. Déby - un rendez vous plusieurs fois repoussé, qui eut lieu finalement début décembre 2012 à Paris. Se rendre ainsi indispensable à la France constitue pour I. Déby une solide assurance pour la suite.

Last but not least, la nature du contingent tchadien n'est pas sans importance. Si peu d'informations circulent sur ce point, il semblerait que l'essentiel des troupes envoyées au Mali appartiennent à la DGSSIE ${ }^{19}$, qui joue au sein de l'armée tchadienne le rôle habituel dévolu aux gardes présidentielles en Afrique (Grilhot, 2013). Composées en grande partie de membres de l'ethnie du président ou de groupes alliés, ces troupes sont les mieux équipées et les mieux traitées par le pouvoir. L'efficacité militaire de cette armée en grande partie milicianisée a déjà été observée à maintes reprises au cours des conflits des deux dernières décennies (Debos, 2013a). Enfin, ces troupes seraient commandées par Mahamat Idriss Déby Itno, un des fils du président, jusque-là plutôt discret (Le Monde AFP, 2013). Nul besoin d'être fin politique pour saisir la signification d'une telle mise en avant dans un contexte tchadien où la légitimité du pouvoir se conquiert à la pointe du fusil - alors que la question de la succession est de celle dont le président use avec brio depuis son arrivée au pouvoir pour contrôler la scène politique n'djaménoise. Dans ce contexte, la dénonciation d'un présumé complot, le $1^{\mathrm{er}}$ mai 2013, suivie d'une vague d'arrestations d'hommes politiques, de hauts fonctionnaires et de journalistes, correspond sans doute moins à une occasion saisie par le pouvoir grisé par ses succès extérieurs pour se débarrasser d'ennemis intérieurs avérés qu'à la pérennité du mode de gouvernement national, dans un jeu politique qui instrumentalise ce type de tension occasionnelle pour resserrer les rangs autour du pouvoir et se perpétuer (Debos, 2013b ; Marchal, 2013). 


\section{Conclusion}

L'intervention militaire tchadienne au Mali s'inscrit donc dans une histoire longue du métier des armes au Tchad et de proximité militaire avec la France. Si l'ère pétrolière a rendu possible la reprise de politiques publiques de développement, et, paradoxalement, ouvert des espaces de débat démocratique au sein de la société, la réponse tchadienne à la crise malienne a rappelé cette réalité nationale inscrite dans la longue durée : la prééminence des enjeux stratégique et de sécurité, garante de légitimité politique et de soutien international. Envoyer l'élite de l'armée gagner de l'argent et des honneurs au Mali - au prix de pertes plus couramment acceptées dans la société tchadienne que dans les sociétés occidentales contemporaines - est un moyen de l'occuper, tout en s'assurant de sa fidélité, en préparant éventuellement une succession dynastique, et en créant une dette à la France. Par son intervention militaire au Mali, le Tchad apporte ainsi une réponse embarrassante au discours de François Hollande ${ }^{20}$ sur la dette de sang contractée par la France envers l'Afrique pendant la Deuxième Guerre mondiale, en l'actualisant. On pressent bien que tout cela ne fait pas l'affaire de la démocratie et du développement, qui risquent de rester des enjeux secondaires. Car une guerre de cette nature, sous bénédiction internationale, dont la rente viendra s'ajouter à celle du pétrole, ne semble pas à même de favoriser la construction d'un État de droit moderne fondé sur le contrôle territorial, la fiscalité et la responsabilité politique devant les citoyens.

\section{BIBLIOGRAPHIE}

Baruch J., 2013. Qui soutient la France au Mali ?. Le Monde, 30 janvier 2013.

Bayart J.F., 2013a. Chronique d'une faillite programmée au Mali. Le Monde, 22 janvier 2013.

Bayart J.F., 2013b. Mali : le choix raisonné de la France. Le Monde, 23 janvier 2013.

Bazenguissa-Ganga R., 1996. Milices politiques et bandes armées à Brazzaville. Enquête sur la violence politique et sociale des jeunes déclassés. Les Études du CERI, $\mathrm{n}^{\circ} 13$.

Bégin-Favre J., 2008. Insécurités. Une interprétation environnementale de la violence au Ouaddaï (Tchad oriental). Thèse de doctorat de géographie, Université de Paris 1, novembre 2008, 2 tomes, 434 p.

Besnier S., 2011. Moussoro. Cent ans déjà. Saint Maur des Fossés, Sépia-PMCT.

Bourgeot A., 2011. Sahara de tous les enjeux. Hérodote n¹42, Géopolitique du Sahara : p. 42-77.

Buijtenhuis R., 1978. Le FROLINAT et les révoltes populaires du Tchad (1965-1976). Den Haag, Paris, New York, Mouton, $526 \mathrm{p}$.

Buijtenhuis R., 1987. Le FROLINAT et les guerres civiles du Tchad. Paris, Khartala, $479 \mathrm{p}$.

Caron L., 2005. Leclerc et le Tchad. Paris, Conférence PMCT.

Caron L., 2009. Au sahara Tchadien. L'administration militaire au moment de l'indépendance Borkou-

Ennedi-Tibesti (1955-1963). Paris, L'Harmattan, 224 p. 
Chauveau J.P., 2005. Les rapports entre générations ont une histoire. Accès à la terre et gouvernementalité locale en pays gban (Centre-Ouest de la Côte d'Ivoire). Afrique contemporaine, $\mathrm{n}$ - 214, p. 59-83.

Darcourt P., 1999. Tchad - Le chemin de la liberté. N’Djaména, Éditions du Chari, 358 p.

De Jong F., Foucher V., 2010. La tragédie du roi Abdoulaye. Néomodernisme et Renaissance africaine dans le Sénégal contemporain. Politique africaine ${ }^{\circ}{ }^{\circledR 118}$, juin 2010, p. 187-204.

Debos M., 2008. Les limites de l'accumulation par les armes. Itinéraires d'ex-combattants au Tchad », Politique africaine ${ }^{\circ} 109$, mars 2008, p. 167-181.

Debos M., 2009. Des combattants entre deux guerres. Sociologie politique du métier des armes au Tchad. Doctorat de science politique, IEP de Paris, $469 \mathrm{p}$.

Debos M., 2013a. Le métier des armes au Tchad : le gouvernement de l'entre-guerres. Paris, Karthala, coll. Les Afrique, $246 \mathrm{p}$.

Debos, 2013b. Pendant la guerre au Mali, au Tchad... Libération, 28 mai : http://www.liberation.fr/ monde/2013/05/28/pendant-la-guerre-au-mali-au-tchad_906388

Garbit F., 1996. Carnets de route d'un méhariste au Tchad (1936-1940). Saint-Maur-des Fossés, Sépia, $198 \mathrm{p}$.

Grégoire E., Bourgeot A., 2011. Désordre, pouvoir et recomposition territoriale au Sahara. Hérodote $n^{\circ}$ 142, Géopolitique du Sahara, p. 3-11.

Grilhot G., 2013. Les forces tchadiennes au Mali : mythe et réalités ", RFI, 21 janvier : http:// www.rfi.fr/afrique/20130119-forces-tchadiennes-mali-mythes-realites-armee-militaire Guengant J.P., 2012. Population, développement et dividende démographique au Tchad. Replacer la population au centre des politiques de développement. Ndjaména, AFD, IRD, $124 \mathrm{p}$.

Guibert N., 2013. Le retour à des options stratégiques plus classiques rassure les militaires français. Le Monde, 17 janvier 2013.

Hervieu S., 2013. Boko Haram : une guerre à huis clos au Nigeria. Le Monde, 5 juin 2013.

Hicks C., 2013. Chad plays politics over costly Mali deployment. African Arguments, 18 avril : http://africanarguments.org/2013/04/18/chad-plays-politics-over-costly-mali-deployment-byceleste-hicks/

Huffington Post, 2013. Le bilan humain au Mali près de deux mois après l'intervention française. Paris, 5 mars 2013.

ICG (International Crisis Group), 2007. République Centrafricaine. Anatomie d'un État fantôme. ICG, Rapport Afrique $\mathrm{n}^{\circ} 136,13$ décembre 2007, 49 p.

ICG (International Crisis Group), 2011a. Le Nord-ouest du Tchad : la prochaine zone à haut risque? ICG, Briefing Afrique $\mathrm{n}^{\circ} 78$, Nairobi, Bruxelles, février 2011, 20 p.

ICG (International Crisis Group), 2011b. La crise libyenne vue de N'Djamena. Thierry Vircoulon, On the African Peacebuilding Agenda, 8 Juin 2011 : http://www.crisisgroup.org/fr/regions/afrique/ afrique-centrale/tchad/op-eds/La\%20crise\%20libyenne\%20vue\%20de\%20NDjamena.aspx

Iyebi-Mandjek O., 2008. Pourquoi les rebelles ont-ils échoué dans la prise de Ndjaména? Yaoundé, Enjeux n³6, juillet 2008.

Khayar I., 1976. Le refus de l'école. Contribution à l'étude des problèmes de l'éducation chez les musulmans du Ouaddaï (Tchad). Paris, Maisonneuve, $140 \mathrm{p}$. 
Lecocq B., 2010. Disputed Desert. Decolonisation, Competing Nationalisms and Tuareg Rebellions in Northern Mali. Afrika-Studiecentrum series, Leiden, Brill.

Lemarchand R., 1986. Chad : The misadventures of the North-South dialectic. African Studies Review, Vol. 29, n 3, septembre 1986, p. 27-41.

Magrin G., 2001. Le sud du Tchad en mutation. Des champs de coton aux sirènes de l'or noir. Paris, SépiaCIRAD, 2001, $427 \mathrm{p}$.

Magrin G., 2008. Tchad 2008. Géographie d'une guerre ordinaire. EchoGéo, n 4, mars-mai 2008, Sur le vif 2008, mis en ligne le 13 mars 2008. URL : http://echogeo.revues.org/document2249.htm 1

Magrin G., 2013. Voyage en Afrique rentière. Une lecture géographique des trajectoires du développement. Publications de la Sorbonne, coll. Territoires en mouvements (à paraître, septembre).

Mahamat Adoum Doutoum, 1994. Actualité tchadienne 1991-1994: Chronique de la Conférence Nationale Souveraine du Tchad (CNS). Processus de démocratisation et résurgence des sectes intégristes au Tchad. Paris, conférence PMCT.

Maoundonodji G., 2009. Les enjeux géopolitiques et géostratégiques de l'exploitation du pétrole au Tchad. Thèse de doctorat en sciences politiques et sociales, Université catholique de Louvain, janvier, 2009,505 p.

Marchal R., 2006. Tchad/Darfour : vers un système de conflits. Politique africaine, $\mathrm{n}^{\circ} 102$, juin 2006, p. $135-154$.

Marchal R., 2013. Le Tchad entre deux guerres? Remarques sur un présumé complot. Politique africaine, $\mathrm{n}^{\circ} 130$.

Musila C., 2012. L'insécurité transfrontalière au Cameroun et dans le bassin du lac Tchad. Paris, Note de l'IFRI, juillet, $32 \mathrm{p}$.

Passages, 2011. Sauver le lac Tchad. Passages, $n^{\circ} 166, \mathrm{n}^{\circ}$ special, $1^{\mathrm{er}}$ trimestre 2011.

Reyna S.P., 1990. Wars without End: a political economy of Bagirmi. Department of Sociology and Anthropology, University of New Hampshire, Durham, 278 p.

Richards P., 1996. Fighting for the Rain Forest. War, Youth and Resources in Sierra Leone. Londres, The International African Institute, Oxford, James Currey, Portsmouth, Heinemann.

Saïbou Issa, 2010. Les coupeurs de route. Histoire du banditisme rural et transfrontalier dans le bassin du lac Tchad. Paris, Karthala.

Saïbou Issa, 2013. Interview du professeur Saibou Issa. Mutations, Yaoundé, 25 février 2013.

Scheele J., 2011. Circulations marchandes au Sahara : entre licite et illicite. Hérodote $\mathrm{n}^{\circ} 142$, Géopolitique du Sahara, $3^{\mathrm{e}}$ trimestre 2011, p. 143-162.

Soares de Oliveira R., 2007. Oil and Politics in the Gulf of Guinea. London, Hurst \& Company, 379 p.

Tubiana J., 2011. Laisser tomber les rebelles. Dimensions locales et régionales du rapprochement TchadSoudan. Genève, Small Arms Survey, 108 p.

Tulipe S., 2008. Tchad : lettre à M. Sarkozy au sujet de la crise et du rôle de la France. Mouvements, 10 février 2008, http://www.mouvements.info/spip.php?article257

Vlassenroot K., 2006. A Societal View on Violence and War. Conflict and Militia Formation in Eastern Congo. In Kaarsholm P. (ed.), Violence, political culture and development in Africa, Oxford, James Currey, p. 49-65. 


\section{NOTES}

1. Al Qaida au Maghreb islamique (Aqmi), le Mouvement pour l'unicité et le djihad en Afrique de l'Ouest (Mujao) et Ansar Dine ( « défenseurs de la religion ») ont des origines diverses. Le premier est directement issu des GSPC (Groupes salafistes pour la prédication et le combat) algériens ; le second rassemble plutôt des ressortissants d'Afrique de l'Ouest (Mauritaniens, Maliens ou Nigériens), le troisième des touaregs maliens dissidents du Mouvement national de libération de l'Azawad (MNLA). Ces mouvements regroupent des doctrinaires religieux et des rebelles, contrebandiers et trafiquants du désert, lesquels y trouvent une légitimation commode et / ou une couverture à leurs activités.

2. La prise des villes du Septentrion malien, marquant la défaite de l'armée nationale malienne face au MNLA, a été l'événement déclencheur d'un coup d'État mené par des officiers subalternes à Bamako, chassant du pouvoir le président Amani Toumani Touré (ATT) peu avant des élections auxquelles il ne se présentait pas. Puis le MNLA a été marginalisé par ses alliés (Aqmi, Mujao, Ansar Dine) qui ont pris le contrôle du Nord Mali.

3. Le 23 mai 2013, le Mujao et «les signataires par le sang» de Mokhtar Belmokhtar revendiquaient le double attentat perpétré au Niger contre l'armée (à Agadez) et Areva (à Arlit).

4. La Cedeao regroupe l'ensemble des pays de l'Afrique de l'ouest du Sénégal au Nigeria. Elle s'est notamment affirmée comme cadre de mobilisation de forces de maintien de la paix (ECOMOG groupe de surveillance de la CEDEAO) en réponse aux crises de la Sierra Leone et du Liberia au cours des années 1990. Le Tchad a été admis comme observateur en ... 2012, dans un contexte marqué par la dégradation de la situation au Mali et au nord-est du Nigeria qui lui est frontalier.

5. Hormis des rébellions Touareg durant les années 1960 et 1990 (voir Lecocq, 2010), qui contribuèrent tout de même aux coups d'État de 1968 et 1991, correspondant à la prise de pouvoir puis à la chute de Moussa Traoré. Après 1991 se mit en place une démocratie volontiers présentée comme modèle par les chancelleries occidentales.

6. Voir l'interview d'Idriss Déby diffusée le 14 avril 2013 sur TV5 Monde (réalisée par Charlotte Bozonnet, Xavier Lambrechts (TV5 Monde) et Bruno Daroux (RFI)).

7. Le fait d'arme le plus marquant est le raid lancé le 5 septembre 1987 par 2000 combattants tchadiens contre la base aérienne libyenne de Maaten As-Sarra : 1713 soldats libyens tués, 300 prisonniers, 70 chars, 30 blindés et 26 avions détruits, tout comme les deux pistes et 8 stations radar (Darcourt, 1999). Ce désastre contraindra le colonel Kadhafi à négocier.

8. Les Toubou du sud sont souvent appelés Gorane par les autres ethnies. Hissein Habré appartient au groupe Anakaza.

9. Un tiers de troupes mises hors de combat à la suite du premier engagement.

10. Mission interafricaine de surveillance des accords de Bangui.

11. L'escadron blanc (1931), Le chef à l'étoile d'argent (1933), La légende du goumier Saïd (1950)...

12. À la Minurcat (Missions des Nations unies en République Centrafricaine et au Tchad) s'ajouta l'Eufor (European Union Force), première opération militaire européenne de cette envergure, sous forte influence française.

13. Les troupes qui ont défendu I. Déby jusqu'au bout en 2008 étaient en grande partie des membres de son clan, alors que l'armée napoléonienne regroupait un grand nombre de nationalités européennes.

14. Rétablissement des capacités africaines de maintien de la paix. Pour en savoir plus : http:// www.un.int/france/frame_francais/france_et_onu/france_et_maintien_de_la_paix/ recamp.htm\#ReCAMP(titre) 
15. À la suite desquels des responsables de l'opposition politique démocratiques seront arrêtés, et où Ibni Oumar Mahamat Saleh, un des principaux leaders, trouvera la mort.

16. La chute du régime Kadhafi en Libye montre cependant les limites d'une telle lecture.

17. Cependant, fin juin 2012 puis en début mai 2013, deux énièmes tentatives de coup d'État fomentées par des proches du régime auraient été déjouées.

18. Qui est lui vice-président de l'Internationale socialiste.

19. Direction Générale des Services de Sécurité et des Institutions de l'État.

20. Cette rhétorique a notamment été utilisée dans le discours prononcé à Bamako le 2 février 2013.

\section{RÉSUMÉS}

Ce texte analyse les raisons de l'intervention militaire tchadienne au Mali début 2013, alors que le Tchad n'est pas un pays voisin du Mali et qu'il n'est pas membre de la Cedeao. Il montre que la raison invoquée par les autorités tchadiennes - éviter la contagion islamiste au Sahel - est secondaire. Cette intervention militaire tchadienne s'inscrit dans une histoire longue marquée par le métier des armes et un compagnonnage stratégique ambigu avec la France. Elle permet à la fois le renouvellement d'une rente diplomatique fort utile dans une région en crise, l'affirmation d'un État renforcé par ses revenus pétroliers, et de resserrer les rangs autour d'un régime structurellement fragile. Cependant, cette aventure militaire risque de compromettre les efforts développementalistes récemment engagés.

This paper analyses the rationale behind the Chadian military intervention in Mali in 2013, which Chad is not a neighboring country, and whereas Chad is not an Ecowas member. We argue that the official reason - preventing Islamist rise into the Sahel - is a secondary one. This Chadian armed intervention takes place in a long soldiering history influenced by a tied but ambiguous relationship with France. It makes possible both to renew a diplomatic rent very useful in a regional disrupt context, to show the regional leadership of the new Chadian petro-State, and to reinforce a regime which stays vulnerable. However, this military adventure could jeopardy the recent efforts to build a more developmental State in Chad.

\section{INDEX}

Keywords : Chad, Mali, French Army, military intervention, soldiering, diplomatic rent, oil context

Mots-clés : Tchad, Mali, armée française, intervention militaire, métier des armes, rente diplomatique, situation pétrolière

\section{AUTEUR}

\section{GÉRAUD MAGRIN}

Géraud Magrin, geraud.magrin@cirad.fr, est chercheur Cirad (UMR Tetis) / UMR Prodig. Il a publié récemment : 
- Losch B., Magrin G., Imbernon J. (dir.), 2013. Une nouvelle ruralité émergente. Regards croisés sur les transformations rurales africaines. Atlas pour le programme Rural Futures du Nepad. Montpellier, Cirad, $46 \mathrm{p}$.

- Magrin G., Raimond C. (dir.), 2012. Atlas de l'Afrique. Tchad. Paris, Les éditions du Jaguar, 135 p. - van Vliet G., Magrin G. (dir.), 2012. Une compagnie pétrolière chinoise face à l'enjeu environnemental au Tchad. AFD, Focales n9, 251 p., novembre 2012 ; http://www.afd.fr/webdav/site/afd/shared/ PUBLICATIONS/RECHERCHE/Scientifiques/Focales/09-Focales.pdf 